Check for updates

Cite this: RSC Adv., 2019, 9, 12346

Received 27th November 2018 Accepted 13th April 2019

DOI: $10.1039 / \mathrm{c} 8 \mathrm{ra0} 09757 \mathrm{~g}$

rsc.li/rsc-advances

\section{The use of fractionated Kraft lignin to improve the mechanical and biological properties of PVA-based scaffolds}

\author{
Petra Rejmontová, ${ }^{\mathrm{ab}}$ Adriana Kovalcik, ${ }^{\text {*cd }}$ Petr Humpolíček, (D) *ab Zdenka Capáková, ${ }^{a}$ \\ Erik Wrzecionko ae and Petr Sáha ${ }^{\text {ab }}$
}

\begin{abstract}
The mechanical properties of poly(vinyl alcohol) (PVA)-based scaffolds were successfully improved. The improvements in mechanical properties correlated with the amount of Kraft lignin in PVA matrices. The critical property for any scaffold is its capacity to allow cells to ingrow and survive within its internal structure. The ingrowth of cells was tested using bioreactors creating simulated in vivo conditions. In the context of all the mentioned parameters, the most advantageous properties were exhibited by the scaffold containing 99 wt\% PVA and 1 wt\% Kraft lignin. The composites with 1 wt\% Kraft lignin exhibited sufficient mechanical stability, a lack of cytotoxicity, and mainly the ability to allow the ingrowth of cells into the scaffold in a rotation bioreactor.
\end{abstract}

\section{Introduction}

The rapidly evolving field of regenerative medicine has created a constant demand for the development of new threedimensional scaffolds with mandatory biological properties (e.g., biocompatibility or a bio-interface) and material properties (e.g., elasticity or porosity). For this purpose, many polymers, natural as well as synthetic, have proven to be suitable matrix materials. Various polymer-based scaffolds have been used for bone, ${ }^{1,2}$ heart, ${ }^{3-6}$ and cartilage ${ }^{7,8}$ regeneration. Particular attention is devoted to, among others, poly(vinyl alcohol) (PVA). This synthetic polymer is already widely utilized in biomedicine. ${ }^{9}$ A wide variety of PVA-based biomaterials have been used for cartilage, ${ }^{\mathbf{1 0 , 1 1}}$ vascular, ${ }^{12}$ cardiovascular, ${ }^{13,14}$ and also bone ${ }^{15}$ tissue replacement. The use of PVA hydrogel as a dermal filler has also been proposed. ${ }^{16}$ The mechanical properties of PVA, however, are not entirely satisfactory. ${ }^{17}$

In contrast to PVA, the biological properties of Kraft lignin (KL) have not yet been satisfactorily described. It is a renewable industrial biopolymer, which has already been tested as an

${ }^{a}$ Centre of Polymer Systems, Tomas Bata University in Zlin, Trida Tomase Bati 5678, 76001 Zlin, Czech Republic. E-mail: humpolicek@utb.cz; Fax: +420-576-031-444; Tel: +420576038035

${ }^{b}$ Polymer Centre, Faculty of Technology, Tomas Bata University in Zlin, Vavreckova 275, 76001 Zlin, Czech Republic

'Institute for Chemistry and Technology of Materials, Graz University of Technology, Stremayrgasse 9, $8010 \mathrm{Graz}$, Austria

${ }^{d}$ Department of Food Chemistry and Biotechnology, Faculty of Chemistry, Brno University of Technology, Purkynova 118, 61200 Brno, Czech Republic. E-mail: adriana.kovalcik@gmail.com

${ }^{e}$ Department of Physics and Materials Engineering, Tomas Bata University in Zlin, Vavrečkova 275, 76001 Zlín, Czech Republic additive and filler for various polymers. ${ }^{18,19}$ The purpose of using $\mathrm{KL}$ as an additive to polymers is mainly to exploit its stiffening effect ${ }^{20}$ and antioxidant efficiency. ${ }^{21}$ Both properties are beneficial for tissue engineering and can be especially advantageous for PVA-based devices. As both inflammatory responses and correlated oxidative stress are typical side effects of implantation, the latter mentioned property of $\mathrm{KL}$ is more than desirable for materials utilized as scaffolds in regenerative medicine. Another favourable feature which KL can bring to the final product is antibacterial activity. However, the antibacterial activity of industrial lignins within polymer composites is questionable. $^{22}$ The antibacterial properties of isolated lignins have been discussed in some recent papers. For example, Medina et al. reported the antibacterial efficiency of hydrolyzed lignin (derived from oil palm empty fruit bunches) against $E$. coli, S. typhimurium, B. subtilis, and $S$. aureus. ${ }^{23}$ Guo et al. reported the antibacterial activity of lignin extracts obtained from anhydrous ammonia-pretreated corn stover after enzymatic hydrolysis. ${ }^{24}$ As KL is a polydisperse compound, its utilization in biomedicine is challenging. However, the fractionation of $\mathrm{KL}$ (e.g., using selective precipitation based on $\mathrm{pH}$ or organic solvent extraction) can improve its polydispersity index and lead to the derivation of specific lignin samples. ${ }^{25}$

In the light of the facts mentioned above, this work aimed to determine the improvement in the function of PVA scaffolds resulting from the incorporation of $\mathrm{KL}$ and the potential of these innovative scaffolds for use in regenerative medicine. Optimally, the novel material should exploit the advantages of lignin, especially its antibacterial activity, excellent mechanical properties, and biocompatibility with PVA. The methanol fraction of Kraft lignin $\left(\mathrm{KL}^{\mathrm{f}}\right)$, obtained according to Gregorova and Sedlarik, ${ }^{26}$ was used to prepare scaffolds. This fraction should 
be more suitable for biomedical applications, as it exhibits lower polydispersity. ${ }^{26}$

\section{Material and methods}

\subsection{Preparation of hydrogels}

Mowiflex TC 232 (Kuraray Europe $\mathrm{GmbH}$ ), a polyvinyl alcohol compound with an aliphatic polyol and calcium distearate, was used as the polymer matrix for hydrogels and is henceforth designated as PVA. Powdered lignin (KL) of the weight average molecular weight $2790 \mathrm{~g} \mathrm{~mol}^{-1}$ and polydispersity of 2.0 was obtained by methanol fractionation of Kraft lignin, which was isolated from black liquor (Zellstoff Pöls AG, Austria) by precipitation with $37 \%$ hydrochloric acid. ${ }^{26}$ The methanol fraction $\mathrm{KL}^{\mathrm{f}}$ was prepared as described by Gregorova and Sedlarik. $^{26}$

$1 \mathrm{~g}$ of PVA was fully dissolved in $20 \mathrm{~mL}$ of Mili-Q water at $80{ }^{\circ} \mathrm{C}$ by stirring in glass vials. Different concentrations of the methanol fraction of $\mathrm{KL}$ were uniformly dispersed in PVA solution. The $\mathrm{pH}$ was adjusted to 2.00 with $2.0 \mathrm{M} \mathrm{HCl}$, and 200 $\mu \mathrm{L}$ of glutaraldehyde was added to the solution and stirred for $30 \mathrm{~min}$. Subsequently, prepared solutions were densified by the repetition of six freeze-thawing cycles (freezing at $-18{ }^{\circ} \mathrm{C}$ for 12 hours followed by thawing (gradual warming) at room temperature for 4 hours). Hydrogels were designated as PVA_X-KL ${ }^{\mathrm{f}}$, where $X$ indicates the concentration of lignin $(1,5,10,15$ and $20 \mathrm{wt} \%)$.

\subsection{Mechanical and thermal stability of hydrogels}

The mechanical stability of fully swollen hydrogels $(15 \mathrm{~mm}$ in diameter and $10 \mathrm{~mm}$ in height) in water was tested by employing creep/creep recovery testing at $25{ }^{\circ} \mathrm{C}$ in air, using a DMA Q800 RH equipped with a submersion compression clamp (TA Instruments, USA). A force of $0.001 \mathrm{~N}, 5$ minutes of isotherm, constant stress of $1 \mathrm{kPa}$ for 5 minutes, and a relaxation time of 5 minutes were applied. Three parallel measurements were performed for each hydrogel.

Thermogravimetric analysis (TG) was performed using STA449C Netzsch equipment. $5 \mathrm{mg}$ of PVA hydrogels were spread in perforated alumina crucibles. Thermal behaviour was recorded from $25{ }^{\circ} \mathrm{C}$ to $550{ }^{\circ} \mathrm{C}$ at a heating rate of $10^{\circ} \mathrm{C} \mathrm{min}{ }^{-1}$ under a nitrogen atmosphere $\left(50 \mathrm{~mL} \mathrm{m^{-1 }}\right.$ of nitrogen flow rate).

\subsection{Internal structure of hydrogels and swelling ratio}

The inner structures of the prepared scaffolds were observed using a Phenom Pro scanning electron microscope (SEM) (Phenom-World BV). The samples were studied at an acceleration voltage of $10 \mathrm{kV}$ in the backscattered electron. Measurements were carried out on samples without prior metallization using a unique sample holder that allows the reduction of charges on nonconductive materials.

The internal structure of hydrogels was determined gravimetrically by estimation of the swelling behaviour in distilled water. The swelling ratio $(Q)$ was calculated by the following equation:

$$
Q=\left(W_{\mathrm{s}}-W_{\mathrm{d}}\right) / W_{\mathrm{d}},
$$

where $W_{\mathrm{d}}$ and $W_{\mathrm{s}}$ are weights of dried hydrogel and swelled hydrogel after 24 hours in water, respectively.

\subsection{Cytotoxicity and cytocompatibility}

The cytotoxicity of scaffolds in direct contact with cells and the cytotoxicity of extracts from scaffolds were tested to reveal the fundamental biological properties of the scaffolds. Accordingly, the materials found to be most cytocompatible were tested for cytocompatibility using bioreactors creating simulated in vivo conditions. This testing allowed the ability of cells to grow into the scaffold's internal pores to be studied. Taken together, the results of the abovementioned tests provided a sophisticated view of the biological properties of the prepared scaffolds based on PVA and KL. All tests were performed using a mouse embryonic fibroblast cell line (ATCC CRL-1658 NIH/3T3, USA). ATCC-formulated Dulbecco's Modified Eagle's Medium (PAA Laboratories $\mathrm{GmbH}$, Austria) containing $10 \%$ bovine calf serum (BioSera, France) and $100 \mathrm{U} \mathrm{mL}^{-1}$ of Penicillin/Streptomycin (GE Healthcare HyClone, United Kingdom) was used as the cultivation medium. The set-ups for individual tests were as follows:

Cytotoxicity in direct contact. Before testing the cytotoxicity of scaffolds in direct contact with cells, samples were disinfected by being submersed in $70 \%$ ethanol. The samples were left in $70 \%$ ethanol overnight; then the ethanol was removed with ultrapure water Milli-Q, which was further changed after 2 hours. The cytotoxicity test in direct contact was performed according to ISO 10993-5 with modifications. The cells were seeded in the presence of samples at a concentration of $10^{5}$ cells per $\mathrm{mL}$. Cell proliferation around the tested samples was evaluated after three days using an inverted Olympus phase contrast microscope (Olympus IX81, Japan). Cells seeded on tissue plastic were used as a reference.

The cytotoxicity of extracts. The test was performed according to ISO 10993-5 and samples were extracted according to ISO 10993-12 (0.2 $\mathrm{g}$ of sample per $\mathrm{mL}$ of cultivation medium). The extraction proceeded at $37 \pm 1{ }^{\circ} \mathrm{C}$ with continuous stirring for $24 \pm 1 \mathrm{~h}$. The individual prepared extracts were then filtered through a $0.22 \mu \mathrm{m}$ syringe filter and utilized within 24 hours. The parent extracts $(100 \%)$ were diluted in the culture medium to obtain a series of dilutions with concentrations of 75, 50, 25, 10 and $5 \%$. Cells were pre-cultivated for $24 \mathrm{~h}$ and the medium was then replaced with diluted extracts. Cells cultivated in the presence of the pure medium were used as a reference (giving $100 \%$ cell proliferation). Viable cells were counted after one day of cell cultivation $\left(37 \pm 0.1{ }^{\circ} \mathrm{C}\right)$ in the presence of sample extracts using a BD FACSCanto flow cytometer (BD Biosciences, Canada) employing SYTO $^{\mathrm{R}} 61$ red fluorescent nucleic acid stain (Life Technologies, USA) to assess cytotoxic effects. First, the diluted extracts were sucked up, and the layer of cells was washed with phosphate buffered saline (PBS, BioSera, France). The adhered cells were released using trypsin and stained with SYTO at a final concentration of $30 \mathrm{nM}$. After 30 minutes, the viable cells were analyzed in the dark. All tests were performed 
in quadruplicates, and Dixon's $\mathrm{Q}$ test was used to remove outlying values. The morphology of the cells was also observed using an inverted Olympus phase contrast microscope (Olympus IX81, Japan).

The ingrowth of cells through the scaffolds. Before testing, samples were disinfected with $70 \%$ ethanol as described earlier. One $\mathrm{mL}$ of cell suspension at a concentration of $1 \times 10^{6}$ cells per $\mathrm{mL}$ was gently injected by syringe into each scaffold [and $0.2 \mathrm{~mL}$ also onto the surface of the material], and the scaffolds were placed in a 24 -well plate. After 2 hours, two $\mathrm{mL}$ of cultivation medium was added. The cells were cultivated in an incubator to allow them to adhere and subsequently began to proliferate inside the structure of the tested samples. After three days, the samples were placed into a bioreactor, where they were cultivated for the next 14 days. For this purpose, a Rotary Cell Culture systems ${ }^{\mathrm{TM}}$ RCCS-4 (Synthecon Incorporated, Texas) was used. Each sample was inserted separately inside the high aspect ratio vessel, and $50 \mathrm{~mL}$ of cultivation medium was added. The forward rotation was adjusted to $15.5 \mathrm{rpm}$. The medium was changed after seven days. After the cultivation period, the samples were fixed with $4 \%$ paraformaldehyde overnight and subsequently washed with PBS, permeabilized with $0.5 \%$ Triton $\mathrm{X}-100$, and again washed with PBS three times. The fixed and permeabilized cells were stained using ActinRed ${ }^{\mathrm{TM}} 555$ (Thermo Fisher Scientific, USA). The tested samples were sliced, and the cell morphology was observed using fluorescent microscopy.

\subsection{Antibacterial properties}

For antibacterial testing, two bacterial strains were utilized Staphylococcus aureus CCM 4516 (ATCC; American Type Culture Collection 6538) and Escherichia coli CCM 4517 (ATCC; American Type Culture Collection 8739). Soybean Casein Digest Agar (Tryptone Soya Agar, HiMedia Laboratories, India) was used as nutrient agar.

To determine whether the amount of $\mathrm{KL}^{\mathrm{f}}$ was sufficient to induce antibacterial properties in the selected scaffolds, the agar diffusion test with modifications was conducted. The amendment lay in the pouring of samples into agar instead of the placing of flat samples onto the surface of the agar. Samples were poured into the agar containing a bacterial suspension at a concentration of $5 \times 10^{5} \mathrm{CFU}$ per $\mathrm{mL}$ of agar. The agar plates with inoculated bacteria were incubated at $35{ }^{\circ} \mathrm{C}$ for 24 hours. After this incubation period, inhibition zones were measured.

\section{Results and discussion}

All biomaterials must exhibit certain required properties and mainly low cytotoxicity. In the case of scaffolds, these include appropriate mechanical properties and advanced properties such as the capacity to allow cells to grow into the structure. Intrinsic antibacterial activity is another advanced and highly desired property which can decrease the risk of nosocomial infections. All of the properties mentioned above were determined for the PVA and $\mathrm{KL}^{\mathrm{f}}$-based scaffolds developed in this study.
PVA hydrogels with the addition of lignin at a concentration higher than $10 \mathrm{wt} \%$ were mechanically unstable and formless. The ability of hydrogels to withstand the mechanical stress was determined as a strain recovery after releasing the stress. Fig. 1 shows the record of creep behaviour of PVA hydrogel modified with $1 \mathrm{wt} \%$ of $\mathrm{KL}^{\mathrm{f}}$.

The maximum strain and strain recovery exhibited unfilled PVA hydrogel (see Table 1). The strain and strain recovery values decreased proportionally with the lignin addition. The reason is that lignin has a reinforcing effect and partially disrupts the homogeneity of the hydrogel. Lignin added at a concentration higher than $10 \mathrm{wt} \%$ resulted in mechanical instability, probably due to the inhibition of PVA cross-linking. Due to this insufficient cross-linking, hydrogels with $15 \mathrm{wt} \%$ and $20 \mathrm{wt} \%$ lignin were soft and formless and therefore unable to recover their original geometry after compression.

PVA_10-KL ${ }^{\mathrm{f}}$ was hydrogel with the highest concentration of $\mathrm{KL}^{\mathrm{f}}(10 \mathrm{wt} \%)$ and still well preserved mechanical stability. Fig. 2 shows the record of thermogravimetric analysis of neat PVA and PVA with $10 \mathrm{wt} \%$ of $\mathrm{KL}^{\mathrm{f}}$. The addition of $10 \mathrm{wt} \%$ of $\mathrm{KL}^{\mathrm{f}}$ shifted the degradation onset about $2.5{ }^{\circ} \mathrm{C}$ to the lower temperature. The reason for an earlier degradation might be a lower crosslinking degree of the hydrogel.

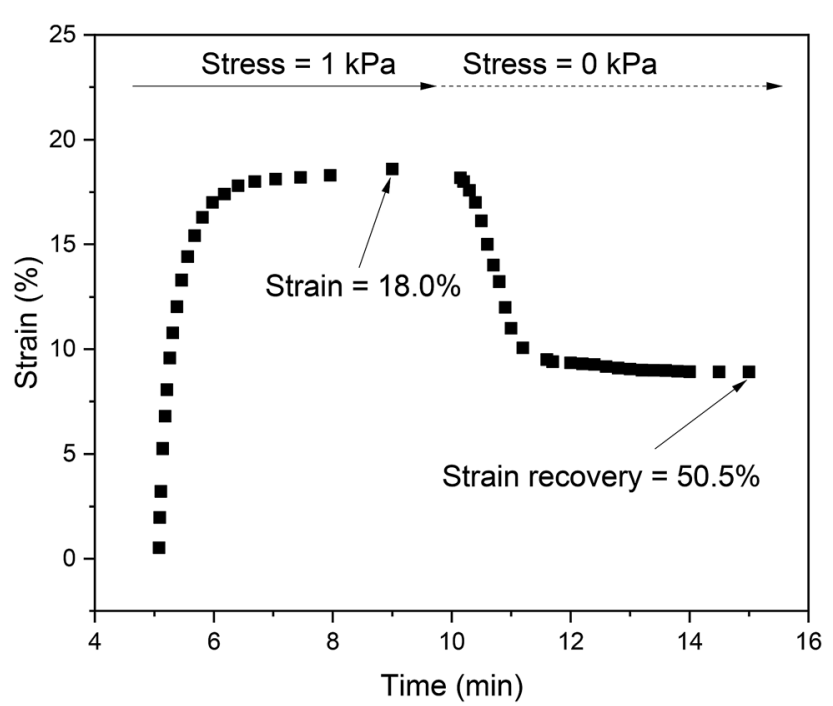

Fig. 1 Creep behaviour of PVA_1-KL ${ }^{f}$ hydrogel at $25^{\circ} \mathrm{C}$ in air.

Table 1 Creep/recovery data for PVA hydrogels

\begin{tabular}{lll}
\hline Sample & $\begin{array}{l}{ }^{a} \text { Compression strain } \\
\text { after creep (\%) }\end{array}$ & $\begin{array}{l}{ }^{b} \text { Strain recovery after 5 } \\
\text { min relaxation (\%) }\end{array}$ \\
\hline PVA & $19.3 \pm 2$ & $54.2 \pm 3$ \\
PVA_1-KL & $18.0 \pm 1$ & $50.5 \pm 3$ \\
PVA_5-KL & $13 \pm 5$ & $45.2 \pm 2$ \\
PVA_10-KL & $8 \pm 1$ & $38.1 \pm 2$
\end{tabular}

${ }^{a}$ The value indicates a reduction in initial sample height due to stress of $1 \mathrm{kPa} .{ }^{b}$ The value indicates the recovery of the original sample height after $5 \mathrm{~min}$ of relaxation without stress. 


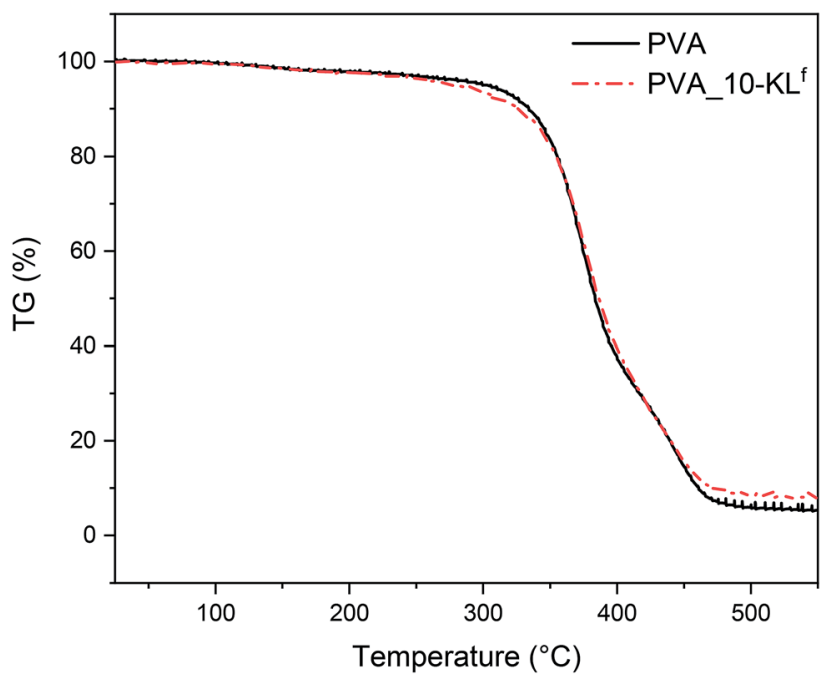

Fig. 2 TG curves of neat PVA hydrogel and PVA hydrogel with 10 wt\% of $\mathrm{KL}^{\mathrm{f}}$.

The internal structures of the tested hydrogels were observed by SEM. The micrographs of PVA (referent sample) and selected samples are depicted in Fig. 3. The micrographs show the structure of PVA/KL ${ }^{\mathrm{f}}$ scaffolds in dependence on the content of
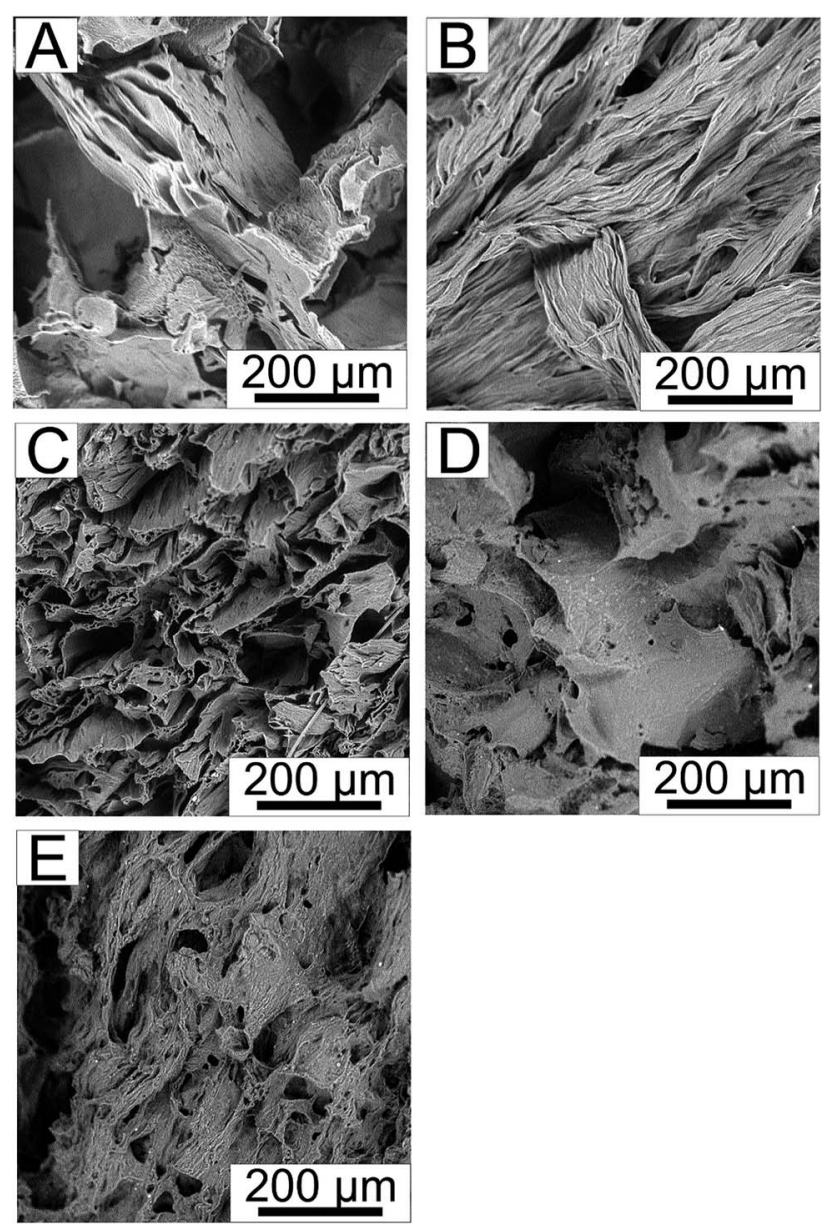

Fig. 3 The internal structure of (A) PVA, (B) PVA_1-KL ${ }^{f}$, (C) PVA_5-KL ${ }^{f}$, (D) PVA_15-KL ${ }^{f}$, (E) PVA_20-KL ${ }^{f}$.
$\mathrm{KL}^{\mathrm{f}}$. The porosity and internal structures of neat PVA and PVA/ $\mathrm{KL}^{\mathrm{f}}$ scaffolds with lower lignin concentrations correspond to structures of scaffolds commonly utilized in tissue engineering. SEM micrographs show that the addition of lignin up to $5 \mathrm{wt} \%$ increased the density of PVA scaffold structures; Fig. 3B shows the longitudinal fibres of PVA_1-KL ${ }^{\mathrm{f}}$, while Fig. 3C depicts the cross-section of PVA_5-KL ${ }^{f}$. However, as the amount of $\mathrm{KL}^{\mathrm{f}}$ increased, the structure became less consistent, as shown in Fig. 3E, which depicts the disruption and mechanical instability of PVA_20-KL ${ }^{\mathrm{f}}$ samples. The observed trend confirmed the changes in hydrogel properties with increasing contents of $\mathrm{KL}^{\mathrm{f}}$ recorded during the testing of mechanical stability.

The differences in the internal structure in dependence on lignin concentration have been confirmed by the values of the swelling ratio (see Fig. 4). The changes in the swelling ratio values are following SEM micrographs. PVA hydrogels with 1$5 \mathrm{wt} \% \mathrm{KL}^{\mathrm{f}}$ are much denser as neat PVA hydrogel and show lower swelling ratio values. However, PVA hydrogels containing lignin in the concentration above $10 \mathrm{wt} \%$ show swelling ratio comparable with neat PVA, which correspond with the content of bigger pores and irregular inner structure.

The determination of cytotoxicity is the test of the first choice when the biocompatibility of any material is at issue. The cytotoxicity testing of scaffolds based on PVA and $\mathrm{KL}^{\mathrm{f}}$ has not yet

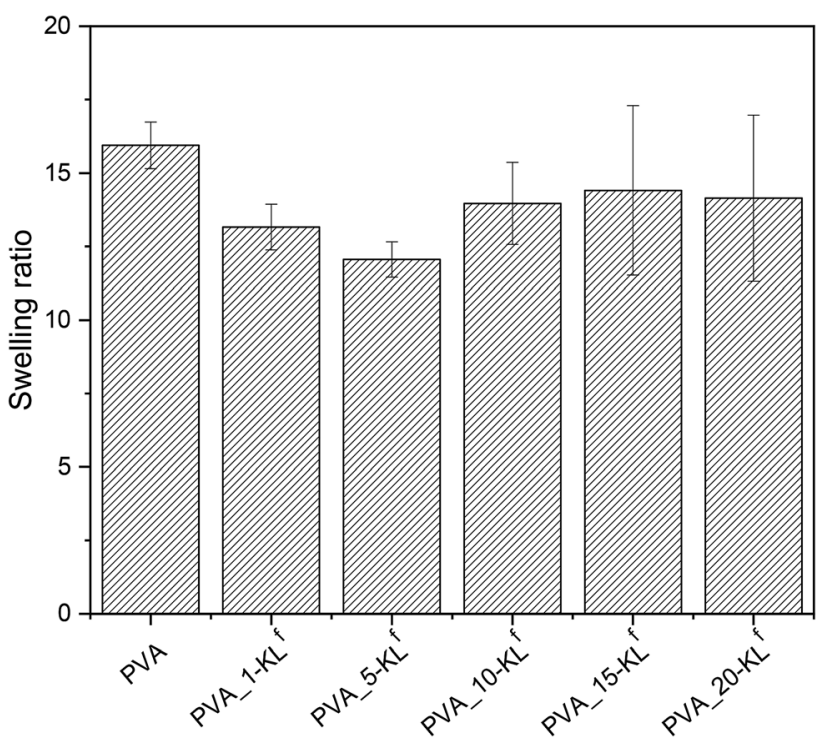

Fig. 4 Swelling ratio of neat PVA and PVA hydrogels modified with $\mathrm{KL}^{f}$.
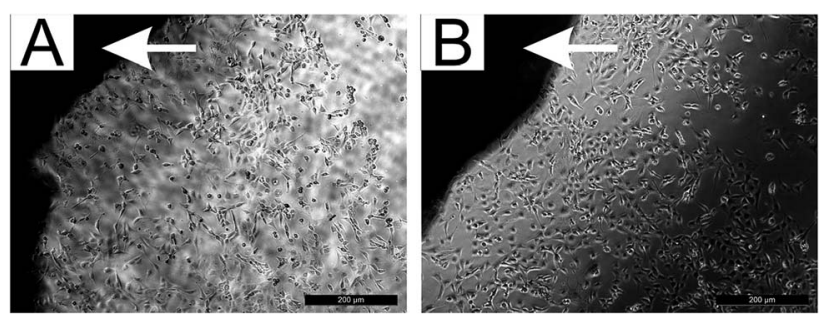

Fig. 5 The mass of $\mathrm{NIH} / 3 \mathrm{T3}$ cultivated in direct contact with (A) PVA_1-KL ${ }^{f}$, (B) PVA_20-KL ${ }^{f}$ (dark regions marked with a white arrow). 
been fully developed. For this reason, a preliminary method for determining scaffold cytotoxicity - that is, cytotoxicity testing in direct contact - was employed first.

It was found that cells were able to proliferate in direct contact with all samples. Moreover, no morphological changes were observed in comparison with reference cells. The obtained results, therefore, indicate no cytotoxic effect in direct contact. The micrographs in Fig. 5 show cells proliferating in direct contact with PVA_1-KL ${ }^{\mathrm{f}}$ and PVA_20-KL ${ }^{\mathrm{f}}$.

For quantitative and more sensitive cytotoxicity assessments, extracts of the studied scaffolds were tested using flow cytometry and SYTO staining. First, the cytocompatibility of pure PVA material in the tested scaffolds, whose biocompatibility has already been described in previous studies, ${ }^{27}$ was confirmed (see Fig. 6, where results for 50, 75 and 100\% extracts are presented; lower extract concentrations exhibited no cytotoxic effect). Concerning the cytotoxicity of scaffold extracts, a significant dependence between the cytotoxic effect and the amount of $\mathrm{KL}^{\mathrm{f}}$ in the tested samples was observed (Fig. 6).

Moreover, cell morphology supported the obtained results, confirming that a direct correlation existed between the cytotoxic effect and the amount of $\mathrm{KL}^{\mathrm{f}}$ in the sample (see Fig. 7).

However, a comparison of the obtained results with the literature is problematic because of a lack of articles dealing
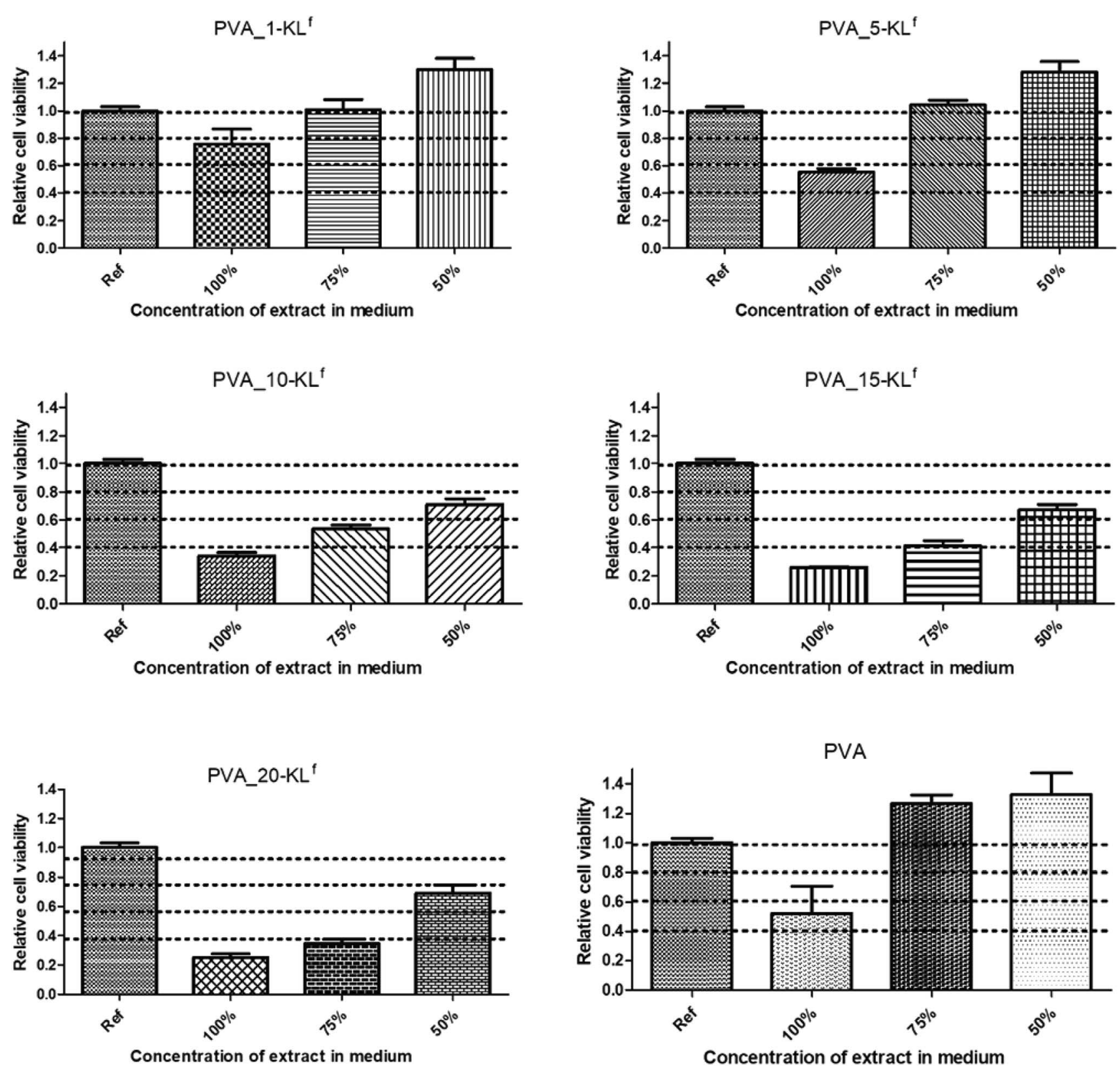

Fig. 6 Cytotoxicity of scaffold extracts of various concentrations presented as a relative number of viable cells \pm the standard deviation compared to reference according to ISO 10993-5 standard. The dashed lines highlight the critical viabilities to be assessed according to requirements of EN ISO 10993-5, where viability > 0.8 means no cytotoxicity; $0.6-0.8$, mild cytotoxicity; $0.4-0.6$, moderate toxicity; and <0.4, severe cytotoxicity. 
with the cytotoxicity and biocompatibility of $\mathrm{KL}$ in general and especially its fractionated form. The obtained results are discussed in the context of more general studies of lignin. Even though the fact that lignin-based copolymers are perceived as nontoxic, ${ }^{28}$ it is necessary to mention that lignin could have an adverse impact on cell viability. The data presented in this article correlate well with the study by Kai $e t a l .{ }^{29}$ They described limitations on the application of alkali lignin resulting from its cytotoxicity at high concentrations. A concentration of $6 \%$ by mass was evaluated as the optimal amount of alkali lignin in PLLA/PLA-lignin nanofibers. ${ }^{29}$ Moreover, it was found that the cytotoxic effect of alkali lignin appeared to increase with time of exposure..$^{30}$ In light of the results obtained in our study, the highest possible concentration of $\mathrm{KL}^{\mathrm{f}}$ that exhibits no or very low cytotoxicity was assessed to be around $5 \%$.

The absence of cytotoxicity is one of the critical factors essential for the successful use of materials in tissue engineering. The ability of cells to ingrow and survive within the internal structure of scaffolds is another crucial parameter. Thus, NIH/3T3 cells were seeded inside the tested samples and cultivated in a Rotary Cell Culture systems ${ }^{\mathrm{TM}}$ RCCS- 4 to evaluate the ability of cells to grow into the porous scaffolds. We found that the cells were able to grow into all the tested scaffolds (see Fig. 8, where only PVA, PVA_1-KL ${ }^{\mathrm{f}}$, PVA_5-KL ${ }^{\mathrm{f}}$, PVA_15-KL ${ }^{\mathrm{f}}$ and PVA_20-KL ${ }^{\mathrm{f}}$ are depicted as examples). However, a significant correlation was found between the ability of cells to grow into scaffolds and the amount of $\mathrm{KL}^{\mathrm{f}}$ present. Greater cell growth into samples with lower amounts of $\mathrm{KL}^{\mathrm{f}}$ was observed, similarly to what was seen during the cytotoxicity testing of extracts. The
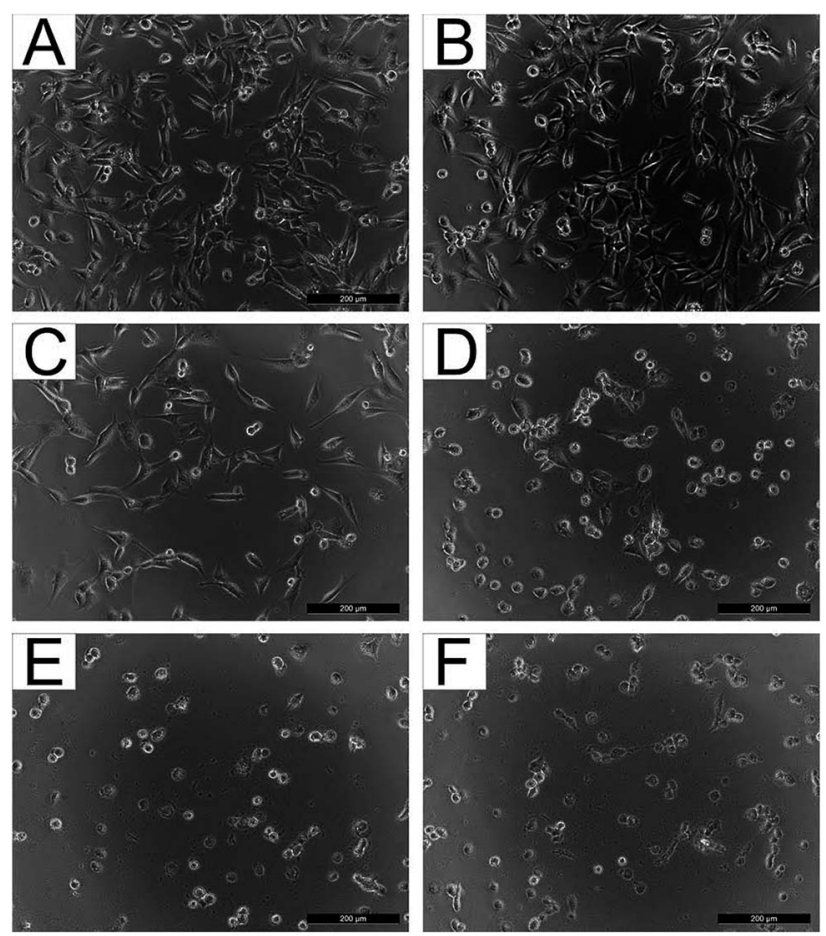

Fig. $7 \mathrm{NIH} / 3 \mathrm{~T} 3$ cultivated in the presence of $75 \%$ extracts of $(A) P V A$, (B) PVA_1- KL ${ }^{f}$, (C) PVA_5-KL ${ }^{f}$, (D) PVA_10-KL ${ }^{f}$, (E) PVA_15- $K L^{f}$, and (F) PVA_20-KL
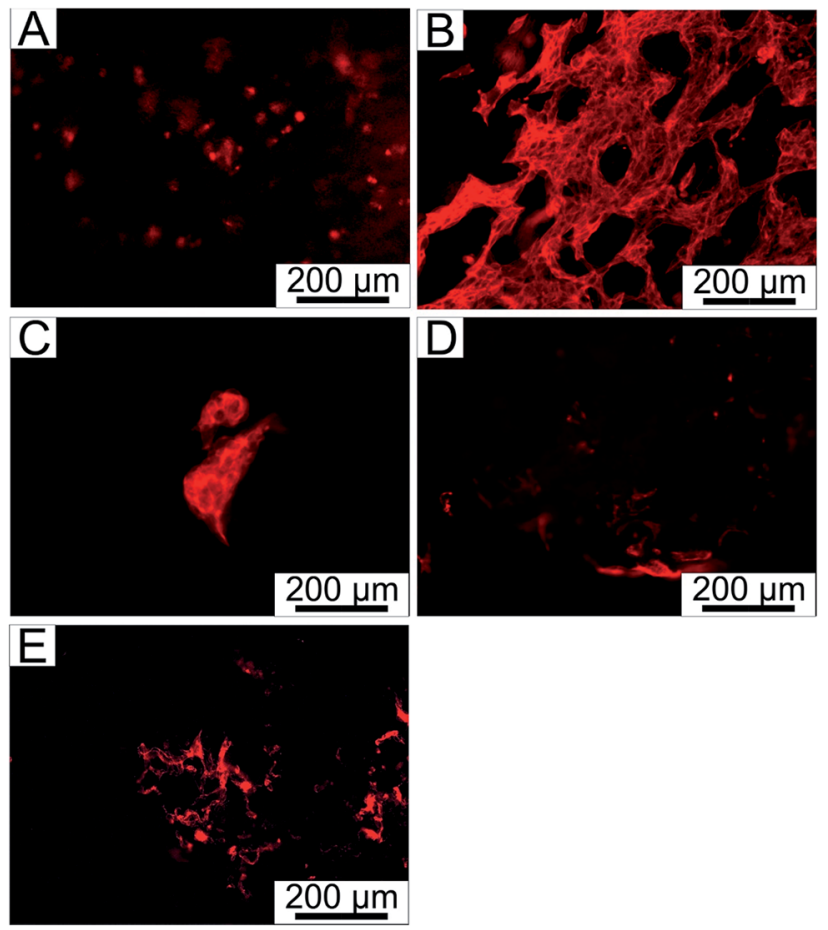

Fig. 8 NIH/3T3 fibroblasts stained with ActinRed ${ }^{T M} 555$ grown within (A) PVA, (B) PVA_1-KL ${ }^{f}$, (C) PVA_5-KL ${ }^{f}$, (D) PVA_15-KL ${ }^{f}$, (E) PVA_20-KL

samples PVA_1-KL ${ }^{\mathrm{f}}$ and PVA_5-KL $\mathrm{KL}^{\mathrm{f}}$ are advantageous for mouse fibroblasts; therefore, the suggested amount of $\mathrm{KL}^{\mathrm{f}}$ for tissue engineering scaffolds seems to be between 1 and $5 \%$. However, according to the comparison of stained cell cytoskeletons inside scaffolds, cell growth was homogeneous only in the case of PVA_1-KL ${ }^{\mathrm{f}}$ (Fig. 8B). In contrast, the cells inside the PVA_5-KL ${ }^{\mathrm{f}}$ (Fig. 8C) scaffold created clusters. Growth is an important feature because a tissue-engineering scaffold should enable cells to grow homogeneously into its structure and gradually create continuous tissue. The creation of separate clusters is undesirable. From this point of view, a concentration of $\mathrm{KL}^{\mathrm{f}}$ of about $1 \%$ should be optimal for use in tissue engineering.

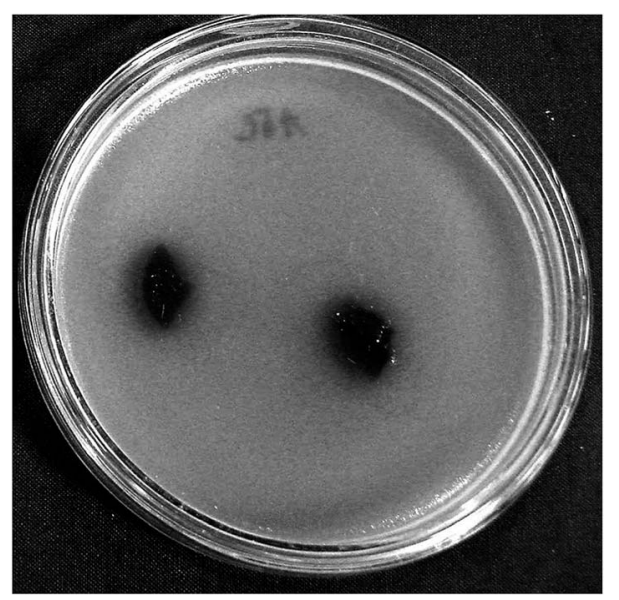

Fig. 9 The growth of $E$. coli in the presence of sample PVA_20-KL ${ }^{\dagger}$. 
To determine whether the amount of $\mathrm{KL}^{\mathrm{f}}$ was sufficient to induce antibacterial properties in the selected scaffolds, the agar diffusion test with modifications was conducted. However, no tested sample exhibited antibacterial properties (see Fig. 9, where no inhibition zones were detected). In this context, it should be mentioned that antibacterial properties of lignins depend on the nature of the lignin and the type of microorganisms. However, the antibacterial efficiency of lignin in polymer composites may be connected with their leaching into the environment. Thus, their antibacterial efficiency in composites in which lignins are chemically crosslinked with other constituents may be limited.

\section{Conclusion}

New scaffolds based on poly(vinyl alcohol) and $\mathrm{KL}^{\mathrm{f}}$ in concentrations ranging from one to twenty wt\% were prepared and biologically tested. Both materials, PVA and $\mathrm{KL}^{\mathrm{f}}$, are biopolymers exhibiting hypothetically high degrees of biocompatibility. $\mathrm{KL}^{\mathrm{f}}$ was used as a stiffening additive with potential antibacterial properties. The determination of viscoelastic properties showed that all scaffolds based on PVA and $\mathrm{KL}^{\mathrm{f}}$ up to $10 \mathrm{wt} \%$ demonstrated sufficient mechanical stability. However, on the basis of advanced biological testing (i.e., according to the ingrowth of cells under simulated in vivo conditions in a bioreactor), it must be noted that only scaffolds containing $99 \mathrm{wt} \%$ PVA and one $\mathrm{wt} \% \mathrm{KL}^{\mathrm{f}}$ can be proposed as advanced materials suitable for use in tissue engineering. Unfortunately, $\mathrm{KL}^{\mathrm{f}}$ incorporated in PVA did not exhibit any antibacterial activity.

\section{Conflicts of interest}

There are no conflicts to declare.

\section{Acknowledgements}

This work was supported by the Czech Science Foundation (grant no. 17-05095S) and the Ministry of Education, Youth and Sports of the Czech Republic (Program NPU I, LO1504). Petra Rejmontová gratefully acknowledges support from the internal grant IGA/CPS/2018/001 of TBU in Zlin, financed from funds for specific academic research. Adriana Kovalcik is grateful for the support through the Brno University of Technology internal project FCH-S-18-5334 and project SoMoPro (project No. 6SA18032). This project has received funding from the European Union's Horizon 2020 research and innovation programme under the Marie Skłodowska-Curie, and it is cofinanced by the South Moravian Region under grant agreement No. 665860. Authors confirm that the content of this work reflects only the author's view and that the EU is not responsible for any use that may be made of the information it contains.

\section{References}

1 B. P. Nair, D. Gangadharan, N. Mohan, B. Sumathi and P. D. Nair, Mater. Sci. Eng., C, 2015, 52, 333-342, DOI: 10.1016/j.msec.2015.03.040.
2 H. Lee, H. Hwang, Y. Kim, H. Jeon and G. Kim, Chem. Eng. J., 2014, 250, 399-408, DOI: 10.1016/j.cej.2014.04.009.

3 M. R. Badrossamay, K. Balachandran, A. K. Capulli, H. M. Golecki, A. Agarwal, J. A. Goss, H. Kim, K. Shin and K. K. Parker, Biomaterials, 2014, 35, 3188-3197, DOI: 10.1016/j.biomaterials.2013.12.072.

4 H. G. S. Ayaz, A. Perets, H. Ayaz, K. D. Gilroy, M. Govindaraj, D. Brookstein and P. I. Lelkes, Biomaterials, 2014, 35, 85408552, DOI: 10.1016/j.biomaterials.2014.06.029.

5 S. Hinderer, J. Seifert, M. Votteler, N. Shen, J. Rheinlaender, T. E. Schaeffer and K. Schenke-Layland, Biomaterials, 2014, 35, 2130-2139, DOI: 10.1016/j.biomaterials.2013.10.080.

6 S. Fleischer, R. Feiner, A. Shapira, J. Ji, X. Sui, H. D. Wagner and T. Dvir, Biomaterials, 2013, 34, 8599-8606, DOI: 10.1016/ j.biomaterials.2013.07.054.

7 S. J. Bryant and K. S. Anseth, J. Biomed. Mater. Res., Part A, 2003, 64, 70-79, DOI: 10.1002/jbm.a.10319.

8 P. J. Martens, S. J. Bryant and K. S. Anseth, Biomacromolecules, 2003, 4, 283-292, DOI: 10.1021/ bm025666v.

9 T. S. Gaaz, A. B. Sulong, M. N. Akhtar, A. A. H. Kadhum, A. B. Mohamad and A. A. Al-Amiery, Molecules, 2015, 20, 22833-22847, DOI: 10.3390/molecules201219884.

10 M. I. Baker, S. P. Walsh, Z. Schwartz and B. D. Boyan, J. Biomed. Mater. Res., Part B, 2012, 100, 1451-1457, DOI: 10.1002/jbm.b.32694.

11 J. C. Hayes and J. E. Kennedy, Mater. Sci. Eng., C, 2016, 59, 894-900, DOI: 10.1016/j.msec.2015.10.052.

12 N. Alexandre, J. Ribeiro, A. Gaertner, T. Pereira, I. Amorim, J. Fragoso, A. Lopes, J. Fernandes, E. Costa, A. Santos-Silva, M. Rodrigues, J. D. Santos, A. C. Mauricio and A. L. Luis, J. Biomed. Mater. Res., Part A, 2014, 102, 4262-4275, DOI: 10.1002/jbm.a.35098.

13 A. F. Leitao, S. Gupta, J. P. Silva, I. Reviakine and M. Gama, Colloids Surf., B, 2013, 111, 493-502, DOI: 10.1016/ j.colsurfb.2013.06.031.

14 S. Mishra, R. Bajpai, R. Katare and A. K. Bajpai, J. Appl. Polym. Sci., 2006, 100, 2402-2408, DOI: 10.1002/app.23177.

15 C. Shuai, Z. Mao, H. Lu, Y. Nie, H. Hu and S. Peng, Biofabrication, 2013, 5, 015014, DOI: 10.1088/1758-5082/5/ 1/015014.

16 L. Dini, E. Panzarini, M. A. Miccoli, V. Miceli, C. Protopapa and P. A. Ramires, Tissue Cell, 2005, 37, 479-487, DOI: 10.1016/j.tice.2005.09.002.

17 A. Karimi and M. Navidbakhsh, Materials and Technology, 2014, 29, 90-100, DOI: 10.1179/1753555713Y.0000000115.

18 O. Gordobil, R. Delucis, I. Eguees and J. Labidi, Ind. Crops Prod., 2015, 72, 46-53, DOI: 10.1016/j.indcrop.2015.01.055.

19 A. T. R. Sugano-Segura, L. B. Tavares, J. G. F. Rizzi, D. S. Rosa, M. G. Salvadori and D. J. dos Santos, Radiat. Phys. Chem., 2017, 139, 5-10, DOI: 10.1016/j.radphyschem.2017.05.016.

20 A. Naseem, S. Tabasum, K. M. Zia, M. Zuber, M. Ali and A. Noreen, Int. J. Biol. Macromol., 2016, 93, 296-313, DOI: 10.1016/j.ijbiomac.2016.08.030.

21 R. Kaur and S. K. Uppal, Colloid Polym. Sci., 2015, 293, 25852592, DOI: 10.1007/s00396-015-3653-1. 
22 A. Gregorova, in Encyclopedia of Biomedical Polymers and Polymeric Biomaterials, 11 Volume Set, ed. M. Mishra, Taylor and Francis Group, New York, 2015.

23 J. D. Coral Medina, A. L. Woiciechowski, A. Zandona Filho, L. Bissoqui, M. D. Noseda, L. P. de Souza Vandenberghe, S. F. Zawadzki and C. R. Soccol, Ind. Crops Prod., 2016, 94, 630-637, DOI: 10.1016/j.indcrop.2016.09.046.

24 M. Guo, T. Jin, N. P. Nghiem, X. Fan, P. X. Qi, C. H. Jang, L. Shao and C. Wu, Appl. Biochem. Biotechnol., 2017, 184, $1-16$.

25 X. Jiang, D. Savithri, X. Du, S. Pawar, H. Jameel, H. Chang and X. Zhou, ACS Sustainable Chem. Eng., 2017, 5, 835-842, DOI: 10.1021/acssuschemeng.6b02174.
26 A. Gregorova and V. Sedlarik, Desalin. Water Treat., 2016, 57, 10655-10663, DOI: 10.1080/19443994.2015.1036364.

27 A. Kumar and S. S. Han, Int. J. Polym. Mater. Polym. Biomater., 2017, 66, 159-182, DOI: 10.1080/00914037.2016.1190930.

28 D. Kai, Z. W. Low, S. S. Liow, A. A. Karim, H. Ye, G. Jin, K. Li and X. J. Loh, ACS Sustainable Chem. Eng., 2015, 3, 21602169, DOI: 10.1021/acssuschemeng.5b00405.

29 D. Kai, W. Ren, L. Tian, P. L. Chee, Y. Liu, S. Ramakrishna and X. J. Loh, ACS Sustainable Chem. Eng., 2016, 4, 52685276, DOI: 10.1021/acssuschemeng.6b00478.

30 V. Ugartondo, M. Mitjans and M. P. Vinardell, Bioresour. Technol., 2008, 99, 6683-6687, DOI: 10.1016/ j.biortech.2007.11.038. 